\title{
Influence of HPV16 E2 and its localisation on the expression of matrix metalloproteinase-9
}

\author{
SABRINA MÜHLEN ${ }^{1,3}$, ANDREAS BEHREN ${ }^{1,4}$, THOMAS IFTNER ${ }^{2}$ and CHRISTIAN SIMON ${ }^{1}$ \\ ${ }^{1}$ Department of Otolaryngology, Head and Neck Surgery, University Hospital Heidelberg, 69120 Heidelberg; \\ ${ }^{2}$ Division of Experimental Virology, Eberhard Karls University Tuebingen, Tuebingen, Germany
}

Received November 25, 2009; Accepted February 4, 2010

DOI: 10.3892/ijo_00000682

\begin{abstract}
Infection with the high-risk HPV types 16 and 18 is the major cause of cervical cancer and plays a role in the development of certain head and neck and skin cancers. We have previously demonstrated that the Early Protein 2 of the Cottontail Rabbit papillomavirus (CRPV), required for skin carcinogenesis in a rabbit model, is able to induce the expression of a matrix metalloproteinase (MMP-9); a protease known to play a key role in invasion and metastasis. However, as of now we do not understand the underlying mechanism of activation nor relevance for the human system. Here, we report that high-risk human papillomavirus HPV16 E2 similar to our previously reported results on CRPV E2 activates the human MMP-9 promoter predominantly via the MEK1ERK1/2-AP-1-signaling pathway. In addition this activation is associated with a nuclear sub-localisation of HPV16-E2 suggesting a nuclear protein-protein or protein-DNA interaction of E2 as the underlying mechanism of activation.
\end{abstract}

\section{Introduction}

Human papillomaviruses have been shown to be the cause for cervical cancer (1) and are associated with other malignancies such as of the head and neck (2), and skin (3). The serotype most frequently found in HPV-positive cervical and head and neck tumours is HPV16 $(1,2)$.

The papillomavirus E2 protein is a phosphoprotein of approximately $48 \mathrm{kDa}$ and functions as a regulator of virus

Correspondence to: Dr Christian Simon, Department of Otolaryngology, Head and Neck Surgery, The University of Heidelberg, Im Neuenheimer Feld 400, 69120 Heidelberg, Germany

E-mail: csimon1407@aol.com;

christian.simon@med.uni-heidelberg.de

Present address: ${ }^{3}$ Newcastle University, Medical School, Institute for Cell and Molecular Biosciences, Newcastle upon Tyne, NE2 4HH, UK; ${ }^{4}$ Cancer Vaccine Group, Ludwig Institute for Cancer Research Ltd., Heidelberg VIC 3084, Australia

Key words: human papillomavirus 16, E2, matrix metalloproteinase-9, AP-1, extracellular regulated kinase genome replication. It is composed of two domains which are separated by a flexible hinge region (4). The $\mathrm{N}$-terminal part of the protein contains the transactivation domain while the C-terminus is occupied by the DNA-binding domain (DBD) (5) and mediates the interaction of E2 with E1 (6). Dimerized, the DBD recognizes E2-binding sites (E2BSs) consisting of the palindromic sequence $5^{\prime}-\mathrm{AAC}(\mathrm{N})_{6} \mathrm{GTT}-3^{\prime}$ (7). E2 is suggested to be the major regulator of E6 and E7 expression and loss of oncogene regulation can be detected in late stages of cervical cancer due to integration of the viral DNA into the host genome and resulting disruption of the E2 ORF (8). In contrast, in HNSCCs viral DNA is found not integrated in the host genome in approximately $35 \%$ of the cases or both episomal and integrated in $17 \%$ of tumours (9). Overexpression of HPV16 and -18 E2 proteins has been shown to induce cell cycle arrest and apoptosis in cervical carcinoma cell lines (10). Further studies led to the discovery that HPV16 E2 interacts with p53 (11) and may induce apoptosis in a p53-dependent manner (12). High-risk HPV E2 proteins can be detected within both, the nucleus and the cytoplasm due to exportin-1 receptor (CRM1)-dependent nucleo-cytoplasmic shuttling, while low-risk HPV E2 is strictly nuclear. These differences are felt to explain why high- but not low-risk HPV E2 induces apoptosis through caspase- 8 in vitro (13).

The E2 protein of the Cottontail rabbit papilloma virus (CRPV) has previously been demonstrated to play a major role in the development of CRPV dependent skin carcinogenesis (14). This is particularly interesting in light of our recently reported finding that the E2 protein of CRPV (15) has the ability to activate the in vitro expression of matrix metalloproteinase 9 (MMP-9), a protein involved in the degradation and remodelling of the extracellular matrix (ECM). Overexpression of MMP-9 has been detected in many different epithelial cancers including of the cervix (16), head and neck (17), and skin (18). MMP-9 plays an important role in the degradation of cell-cell and cell-matrix molecules, induction of cell migration, and the release and activation of growth factors (19).

We also previously reported that the CRPV-E2-induced expression of MMP-9 is regulated via the transcription factor complex AP-1 (20). AP-1 is a homo- or heterodimeric transcription factor consisting of members of the jun, fos and/or fra family (21) and is a main target of MAPKs (22). MAPKs are components of signal-transduction pathways that consist of protein kinases of which the extracellular regulated kinase 
(ERK), the c-Jun N-terminal kinase (JNK) and the p38 pathway represent the most extensively studied (23). The ERK pathway consists of Raf-, MEK, and ERK-proteins that are sequentially activated through both extrinsic (i.e. EGF, FGF, PDGF and NGF) (23) and intrinsic (cytoplasmic) (i.e. Ras associated with Golgi, PARP-1, p53R2) (2426) signals.

As of now we do not understand the relevance of CRPVE2-dependent activation of MMP-9 for the human system, i.e. whether high-risk HPV-E2 has the same effect on MMP-9. Although we have previously shown that CRPV-E2 requires an activation of MEK-ERK but not p38 and JNK to induce rabbit MMP-9 promoter activity (20), the underlying mechanism of activation remains obscure. Given that nuclear and cytoplasmic proteins activate the MEK-ERK signalling pathway and moreover that the sub-cellular localisation of HPV16 E2 is critical for the execution of E2 dependent phenotypes, i.e. apoptosis (13), we hypothesized that the same may hold true for the induction of MMP-9 expression.

Towards the end of this study we provide evidence that similar to CRPV-E2 human high-risk HPV16 E2 induces MMP-9 expression via MEK1-ERK1/2, AP-1. Moreover, our data suggest that the activation of MMP-9 expression is associated with the intracellular localisation of the viral protein. Our finding that depleting the nucleus from HPV16 E2 significantly reduced MMP-9 promoter activation suggests that a nuclear protein-protein or protein-DNA interaction is relevant for MMP-9 expression.

\section{Materials and methods}

Plasmids. Human MMP-9 promoter (2305 bp) were amplified using PCR to introduce restriction sites $K p n I$ and $B g l$ II to allow for cloning of the promoter fragment into the chloramphenicol acetyltransferase reporter-gene vector pCAT3 Basic (Promega). The mammalian expression vector pCMV-HA (Clontech) was used to construct pCMV-HA HPV16 E2 and pCMV-HA HPV16 E2 eGFP fusion protein plasmids. In both cases the full length HPV16 E2 DNA fragment was amplified by PCR and introduced into the vector. For localisation studies, the HPV16 E2 gene introduced into pCMV-HA was mutated using QuikChange (Stratagene) according to the manufacturer's recommendations and confirming the desired mutations by sequencing. The TAM67 construct has been described elsewhere (27), and the dominant negative ERK constructs ERK1 (K71R) and ERK2 (K52R) which are mutated within their ATP-binding pockets were cloned into pcDNA3 (Invitrogen).

Cell culture, and transient transfections, and preparation of cell lysates. C33A human cervical keratinocytes (ATCC) and NIH3T3 mouse fibroblasts were maintained in Dulbecco's modified Eagle's medium (DMEM) with glutamine (PAA, Linz, Austria) supplemented with $10 \%$ fetal bovine serum (PAA) and 5\% AntibioticAntimycotic (Invitrogen). Cells were grown at $37^{\circ} \mathrm{C}$ and $5 \% \mathrm{CO}_{2}$. Transfection of cells with plasmids was carried out using Lipofectamine ${ }^{\mathrm{TM}}-2000$ (Invitrogen) according to the supplied protocol. Forty-eight hours after transient transfection, cells were harvested and the protein content determined using BCA (BCA kit, Pierce). The lysates were equalized for protein content and used for reporter-gene assays, immunoprecipitation or separated by SDS/PAGE.
RNA isolation with subsequent RT-PCR. The RNA isolation was carried out using the RNeasy kit (Qiagen) according to the manufacturer's protocol. On-column DNA digestion was performed using the RNase free DNA kit from Qiagen. After RNA isolation $1 \mu \mathrm{g}$ RNA was used in a reverse transcription reaction with the high-capacity cDNA kit (Applied Biosystems) as per manual. Primer sequences are as follows: MMP9PhumKpnF: 5'-CAGGTACCTACCCACTTCTATAC CTGGG-3' (KpnI); MMP9PhumKpnR: 5'-GGTACCAGAG GCTCATGGTGAGGG-3' (KpnI); hMP-E2mut-for: 5'-GCA GCTTAGAGCCCAATAACATGGTTTTGTGATTCCAA G-3'; hMP-E2mut-rev: 5'-CTTGGAATCACAAAACCAT GTTATTGGGCTCTAAGCTGC-3'; NLS1 (107/108): 5'-GT GTATTTAACTGCACCAAAACGATGTATAAAAAAAC ATGGATATACAGTGGAAGTGC-3'; NLS1 (107/108) rev: 5'-GCACTTCCACTGTATATCCATGTTTTTTTATACAT CGTTTTGGTGCAGTTAAATACAC-3'; NLS2 (241/244): 5'-CACAGACGACTATCCAGCGAAAAAGATCACGGC CAGACACCGGAAACCCC-3'; NLS2 (241/244)rev: 5'-GG GGTTTCCGGTGTCTGGCCGTGATCTTTTTCGCTGGAT AGTCGTCTGTG-3'; NLSDBDmt: 5'-GATGCTAATACTT TAAAATGTTTAGGATATGGATTTACAAAGCATTGTA CATTGTATACT-3'; NLSDBDmt rev: 5'-AGTATACAA TGTACAATGCTTTGTAAATCCATATCCTAAACATTT TAAAGTATTAGCATC-3'; QPCRMMP9hFor 5'-CGACGT CTTCCAGTACCGA-3'; QPCRMMP9hRev 5'-CTCAGGG CACTGCAGGAT-3'; QPCRACTBhFor 5'-GCACAGAGC CTCGCCTT-3'; QPCRACTBhRev 5'-CCTTGCACATGCCG GAG-3'; QPCRGAPDHhFor 5'-CTCTGCTCCTCCTGTTC GAC-3'; QPCRGAPDHhRev 5'-TTAAAAGCAGCCCTGG TGAC-3'; qPCRHPV16E2 5'-ATGGAGGACTCTTTGCC ACG-3'; qPCRHPV16E2rev 5'-CCTGTTGGTGCAGTTAA ATACAC-3'.

Semi-quantitative PCR. Semi-quantitative PCR was carried out in a BioRad thermocycler (BioRad, Munich, Germany) using $10 \mathrm{ng}$ template cDNA and amplifying for 33 cycles at $60^{\circ} \mathrm{C}$.

Reporter-gene assays. The cell lysates normalized for protein content were subjected to CAT-ELISA (Roche) according to the manufacturer's manual.

Western blotting and immunoprecipitation. After SDS/PAGE proteins were transferred to PVDF membrane for $1 \mathrm{~h}$ at $100 \mathrm{~V}$. Membranes were blocked either in 4\% BSA/TBST overnight. Anti-ERK primary antibody was used at 1:2000 and anti-pERK at 1:1000 (both CST). Primary antibody incubation was conducted at $4{ }^{\circ} \mathrm{C}$ overnight in $5 \% \mathrm{BSA} / \mathrm{TBST}$ on a rotary shaker. An HRP-conjugated secondary antibody was used at 1:5000 for $1 \mathrm{~h}$ at RT followed by ECL plus (GE Healthcare, Chalfont St. Giles, UK) based chemiluminescence detection. p-ELK was detected after immunoprecipitation overnight and subsequent kinase assay according to the manufacturer's protocol using the p44/42 MAP Kinase Assay kit (CST).

DNA staining and immunofluorescent detection of proteins. For nuclear staining prior to microscopic investigation or fixation for immunofluorescence, cells were incubated with either $0.2 \mu \mathrm{g} / \mathrm{ml}$ Hoechst-33342 Dye (Sigma-Aldrich, Munich, Germany) or $1 \mu \mathrm{g} / \mathrm{ml}$ DAPI (Carl Roth GmbH, Karlsruhe, Germany) for $30 \mathrm{~min}$ to $1 \mathrm{~h}$ at $37^{\circ} \mathrm{C}, 5 \% \mathrm{CO}_{2}$. 
A

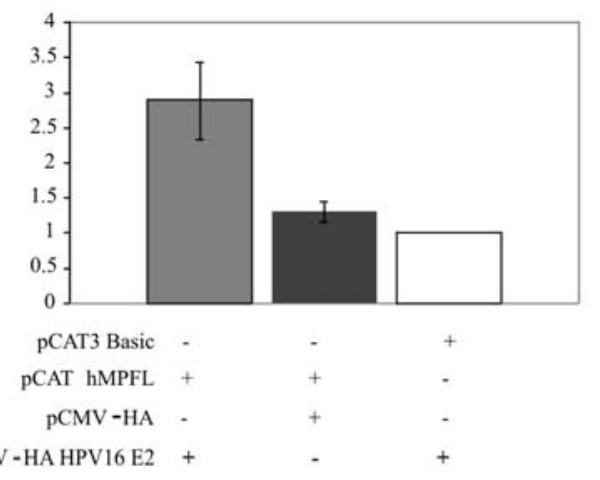

B

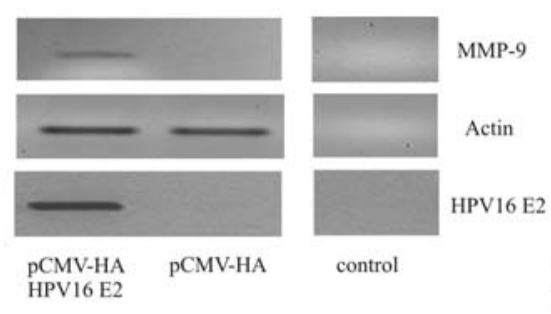

C

- $1202 \mathrm{pp}$ - CCCCAATTTG CAGTTGAAGAATCCTAAGCT GACAAAGGGG AAGGCATTTA CTCCAGGTTA CACTGCAGCT TAGAGCCCAA TA ACCTGGTT TGGTGATTCC AAGTTAGAAT CATGGTCTTT TGGCAGGGTC TCGCTCTGTT GCCCAGGCTG GAGTGCAGTG ACATAATCAT GGCTCACTGT $\quad$ - $\quad$ - 1022bp

Figure 1. Activation of the MMP-9 promoter and MMP-9 transcript upregulation by HPV16 E2. (A) Reporter gene assay of C33A cells transiently transfected with the full length MMP-9 promoter reporter-gene vector, empty control vector, empty expression vector, and HPV16 E2. (B) Relative amount of MMP-9, actin, and HPV16 E2 mRNA transcripts at cycle 33 after transient transfection with indicated constructs, with $\mathrm{H}_{2} \mathrm{O}$ as negative control. (C) Schematic representation of the MMP-9 promoter construct used with the sequence of the E2BS indicated in bold and effect of the E2BS mutation on HPV16 E2-mediated activation as compared to the wild-type promoter. ${ }^{*} \mathrm{P} \leq 0.05,{ }^{* *} \mathrm{P} \leq 0.01$ and ${ }^{* * *} \mathrm{P} \leq 0.005$ as obtained by Students' t-test. Results show mean value out of three experiments performed in triplicate.

For direct immunofluorescence of the HPV16 E2-fusion proteins, the transfected cells were grown on glass coverslips for $24 \mathrm{~h}$, fixed with $4 \%$ paraformaldehyde in phosphatebuffered saline for $15 \mathrm{~min}$ at room temperature. Following fixation, the coverslips were blocked in 1\% BSA in PBST for 30 min. The anti-actin antibody (sc-1616; Santa Cruz Biotechnology, Heidelberg, Germany) was diluted 1:100 in $1 \% \mathrm{BSA}$ in PBST and cells were incubated for $1 \mathrm{~h}$. After incubation with the secondary antibody (sc-2313; Santa Cruz Biotechnology) for $1 \mathrm{~h}$, coverslips were washed with PBS and rinsed in water before transferring them onto slides using VectaShield mounting medium (Linaris, Wertheim, Germany).

Correlation of sub-cellular fluorescence. Imaging software ImageJ was used to determine the correlation between whole cell and nuclear fluorescence after imaging of immunofluorescences. Relative fluorescence ratios were thus determined for an average of 10 transfected cells.

\section{Results}

Firstly, the capacity of HPV16 E2 to activate the MMP-9 promoter was investigated using a reporter-gene vector containing $2305 \mathrm{bp}$ of the human MMP-9 promoter (termed full length) in front of the gene encoding chloramphenicol acetyltransferase (CAT). Cell lysates of C33A cells were obtained to examine the effect of HPV16 E2 on the transcription of MMP-9 from cells transiently co-transfected with HPV16 E2 and the reporter-gene vector were analysed for CAT-expression by ELISA. It could be observed that HPV16 E2 was able to significantly activate the human MMP-9

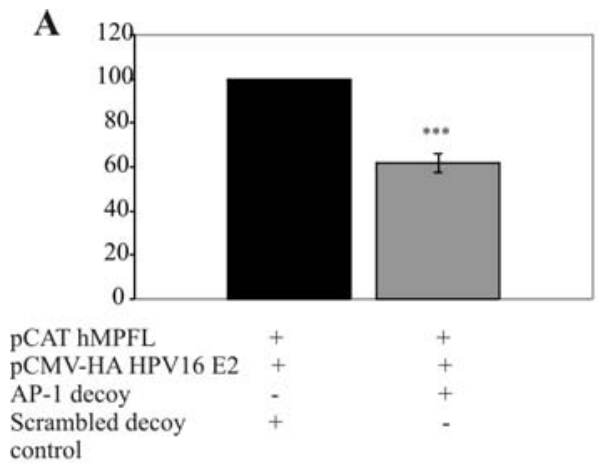

B

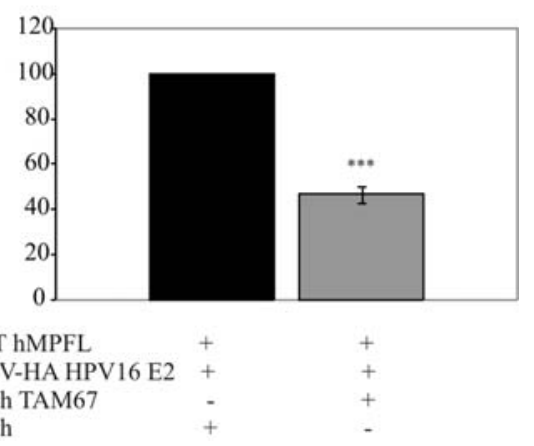

Figure 2. Involvement of AP-1 in the activation of the MMP-9 promoter by HPV16 E2. (A) Effect of transient co-transfection of an AP-1 decoy in reporter-gene assays as compared to the scrambled decoy control, and (B) effect of co-transfection of the transactivation-deficient c-Jun mutant TAM67 along with HPV16 E2 expression plasmid as compared to the empty vector control. ${ }^{*} \mathrm{P} \leq 0.05,{ }^{* *} \mathrm{P} \leq 0.01$ and ${ }^{* * *} \mathrm{P} \leq 0.005$ as obtained by Student's t-test. Results show mean value out of three experiments performed in triplicate. 

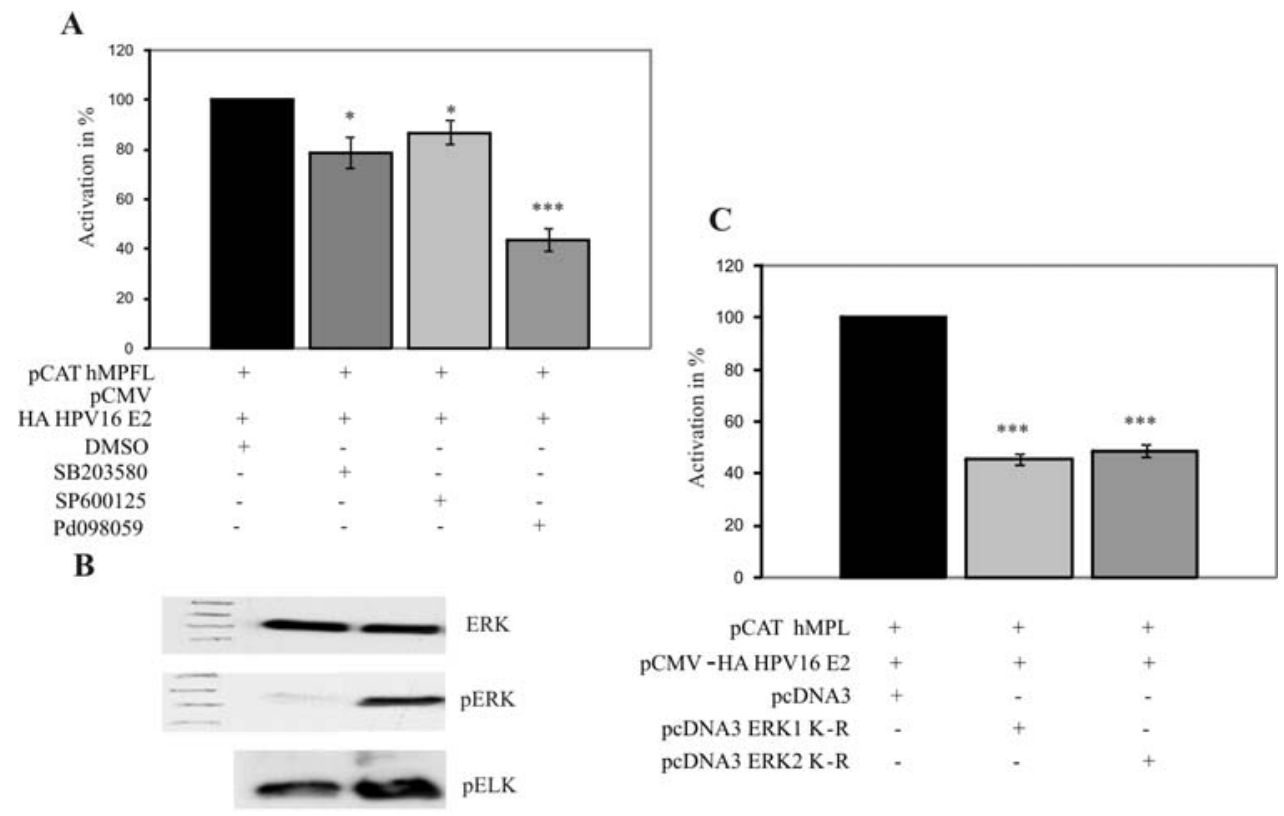

$$
\text { 这 }
$$

Figure 3. Involvement of MAP-kinases in the HPV16 E2-mediated activation of the MMP-9 promoter. (A) Effect of MAP-kinase inhibitor treatment on the activation of the MMP-9 promoter by HPV16 E2, and (B) phosphorylation state determination of ERK and its downstream target ELK after transient transfection of C33A cells with HPV16 E2. (C) The effect of ERK activation on E2-mediated promoter activation as determined by co-transfection with dominant-negative ERK 1 or $2 .{ }^{*} \mathrm{P} \leq 0.05,{ }^{* *} \mathrm{P} \leq 0.01$ and ${ }^{* * *} \mathrm{P} \leq 0.005$ as obtained by Students' t-test. Results show mean value out of three experiments performed in triplicate.

promoter by $3 \pm 0.8$-fold (Fig. $1 \mathrm{~A}$ ). To address the question of up-regulation of the endogenous MMP-9 transcripts, human cervical keratinocytes were transiently transfected with HPV16 E2. After isolation of RNA, RT-PCR was performed. The obtained cDNA was subjected to semi-quantitative PCR where a significant amount of MMP-9 mRNA in pCMV-HA HPV16 E2 transfected cells could be detected (Fig. 1B). These results suggest a relevance of E2-dependent MMP-9 expression in the human system.

Examining the promoter sequence of the human MMP-9 construct, we observed the presence of a binding site for E2 (E2BS; Fig. 1C) at -1120 to -1109 bp in the MMP-9 promoter which is not present in the rabbit MMP-9 promoter recently described by us (20). We hence investigated whether direct binding of HPV16 E2 plays a role in the activation of the promoter by deleting the consensus sequence using site-directed mutagenesis. It could be seen from the results of subsequent reporter-gene assays with the mutant promoter that this E2BS has no influence on the activation of the human MMP-9 promoter by HPV16 E2 (Fig. 1C).

It has been previously described that the binding sites for the transcription factor complex AP-1 are of importance in the activation of the rabbit MMP-9 promoter by CRPV E2 and that the proximal AP-1 binding site in the human promoter is of importance in E2-mediated activation $(15,20)$. Hence, an AP-1 decoy was used in this study to confirm the involvement of the AP-1 complex in promoter activation by HPV16 E2. A decoy is a short synthetic oligonucleotide that resembles the transcription factor consensus sequences and hence competes for binding of the transcription factor with consensus sequences in target genes. Co-transfection of the AP-1 decoy with the human MMP-9 promoter and HPV16 E2 resulted in a decrease in activation by $40 \pm 4 \%$ as compared to the negative control decoy (Fig. 2A).

The transcription factor AP-1 is a dimeric complex which consists of members of the jun-, fos-, and fra-families (21). To determine the importance of the AP-1 complex for activation of the MMP-9 promoter, the transactivation-deficient c-Jun mutant TAM67 (27) was used for co-transfection with the MMP-9 promoter and HPV16 E2. In subsequent reporter-gene assays, the activation of the MMP-9 promoter was shown to be decreased to $46 \pm 4 \%$ when compared to the empty vector control (Fig. 2B).

Expression of the components of the transcription factor complex AP-1 and its activation through phosphorylation is largely regulated by the members of the mitogen-activated protein kinase (MAPK) pathway. In order to investigate the role that MAP-kinases play in the activation of E2-mediated MMP-9 promoter activation, chemical inhibitors of the three main MAPK/SAPK pathways JNK, ERK, and p38 were used in reporter-gene assays. It could be observed that use of the MEK1 inhibitor PD098059 led to a highly significant decrease in the activation potential of the HPV16 E2 protein of about $60 \%$ (Fig. 3A). The p38 inhibitor SB203580 led to a moderate decrease in promoter activation of about $20 \%$, while the JNK inhibitor SP600125 had little effect on the E2-mediated activation of the promoter. Looking at the effect that the ERK inhibitor has on the activation of the MMP-9 promoter, ERK must be phosphorylated in response to E2 expression. Hence, the phosphorylation status of ERK in transfected cells was 
A

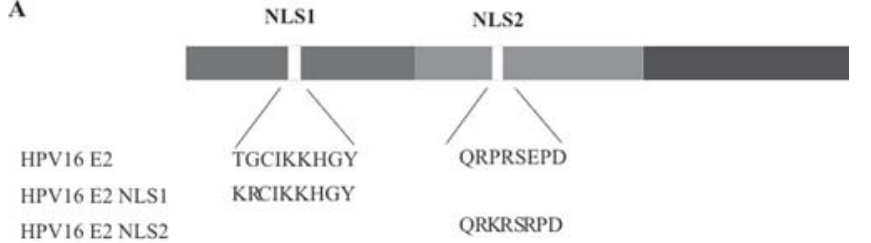

B

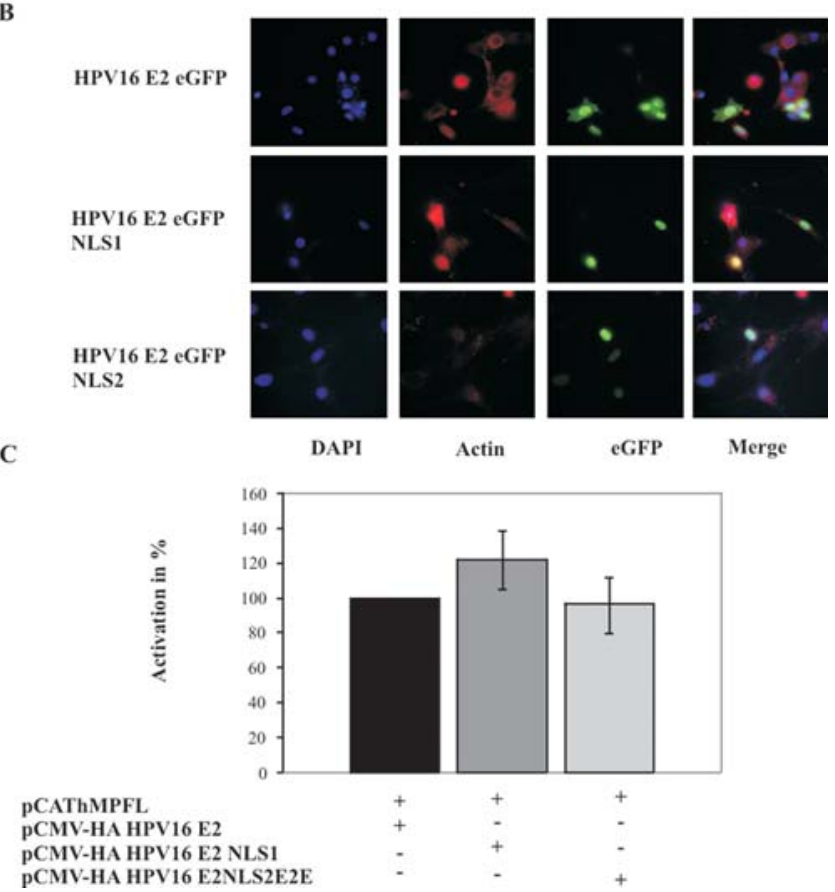

Figure 4. Effect of the nuclear localisation signals of low risk papillomavirus E2 proteins introduced into HPV16 E2. (A) Schematic representation of the mutations introduced into the E2 ORF, (B) and determination of the subcellular localisation of the resulting mutant-eGFP fusion proteins in NIH3T3 cells (blue, DAPI; red, actin; green, eGFP). Pictures were taken as mentioned in Fig. 4. (C) Effect of the mutated HPV16 E2 on the activation of the MMP-9 promoter as compared to wild-type E2 referred to as $100 \%$. ${ }^{*} \mathrm{P} \leq 0.05,{ }^{* *} \mathrm{P} \leq 0.01$ and ${ }^{* * *} \mathrm{P} \leq 0.005$ as obtained by Students' t-test. Results show mean value out of three experiments performed in triplicate.

investigated more closely by immunoprecipitation and Western blotting. As was expected, expression of E2 led to an elevated level of fully functional pERK able to activate an exogenously added downstream-target ELK as shown by in vitro kinase assay as compared to expression of the empty vector control (Fig. 3B).

Further, human cells were transiently co-transfected with the reporter-gene vector, HPV16 E2, and dominant negative mutants of ERK1 and ERK2. Subsequent reporter-gene assays led to the observation that co-expression of either of the two dominant negative mutants had a similar effect which resulted in a reduction of activation of about $50 \%$ as compared to the empty vector control (Fig. 3C).

It has been reported by Blachon et al (13) that high-risk human papillomavirus E2 as opposed to low-risk HPV is able to shuttle between nucleus and cytoplasm. The study related the cytoplasmic localisation of the E2 protein with the induction of apoptosis through a caspase- 8 dependent mechanism by high-risk HPV. Moreover, the MEK1-ERK1/2 signalling pathway can be induced via various activators that can be cytoplasmic proteins (i.e. PARP, p53R2) through direct protein-protein interaction $(26,25)$ or de novo synthesized growth factors (i.e. EGF, FGF) through protein-receptor interaction $(28,29)$. It was hence of interest for the present study whether the localisation of the E2 protein has an impact on its induction of MMP-9 expression.

In order to carry out localisation studies, HPV16 E2 was tagged with eGFP to allow for easy visualisation. The protein also obtained an HA-tag at the $\mathrm{N}$ - and a KT3-tag at the $\mathrm{C}$-terminus to determine the integrity of the protein.

Immunofluorescence revealed that eGFP-HPV16 E2 was present in the nucleus as well as within the cytoplasm (Fig. 4B). It has been described that HPV16 E2 contains only one nuclear localisation sequence (NLS) as compared to HPV6b E2 which has three (13). However, HPV16 E2 has a nuclear export sequence (NES) which is not found in the low-risk type. Hence, different approaches were used to investigate the potential role of E2 localisation in MMP-9 promoter activation. Firstly, in order to increase the amount of nuclear HPV16 E2 either one of the two additional NLS found in HPV6b E2 were introduced to HPV16 E2, resulting in the mutant constructs HPV16 E2 NLS1 and HPV16 E2 NLS2 (Fig. 4A). We decided not to delete the NES found in HPV16 E2, because the position in the N-terminal domain is within the transactivation domain of the protein with the consecutive risk of false-positive results (14). Using immunofluorescence and the eGFP-tagged E2 mutants, the localisation of the mutant proteins was determined.

Immunofluorescence of the NLS mutant HPV16 E2-eGFP fusion proteins revealed that both mutants containing an additional NLS site were localised solely within the nucleus (Fig. 4B). In reporter-gene assays, the ability of these mutant proteins to activate the MMP-9 promoter was investigated. Here, the NLS mutant constructs showed no significant decrease in their activation potential of the MMP-9 promoter (Fig. 4C) suggesting that a cytoplasmatic localisation of E2 is not critical for the induction of MMP-9 promoter activation.

To further investigate this hypothesis, a second mutant was created in which the only NLS found in HPV16 E2 is rendered non-functional (Fig. $5 \mathrm{~B}$ ) resulting in a mainly cytoplasmic localisation of the protein (Fig. 5A and C). When this mutant was used in reporter-gene assays with the human MMP-9 promoter, a $60 \%$ reduction of CAT-expression compared with wild-type HPV16 E2 was observed (Fig. 5D) indicating that a nuclear localisation of HPV16 E2 is significantly elevating MMP-9 promoter activity.

\section{Discussion}

Recently published data indicate that CRPV-E2 is required for the development of skin cancers in a rabbit model and moreover activates the expression of a matrix metalloproteinase highly relevant for epithelial cancer invasion. However, we currently do not know, if this finding translates equally into the human system nor do we understand the underlying mechanism of activation. In this report we demonstrate that similar to CRPV-E2 the human high-risk HPV16 E2 protein induces the expression of MMP-9 through the activation of MEK1-ERK1/2 and AP-1. Moreover, our data suggest that this mechanism relies on a predominantly nuclear sublocalisation of HPV16.

Aberrant expression of c-Jun has been shown to be involved in several human cancers such as myeloid leukaemia, Hodgkin lymphoma, and prostate cancer $(30,31)$. In head and neck 


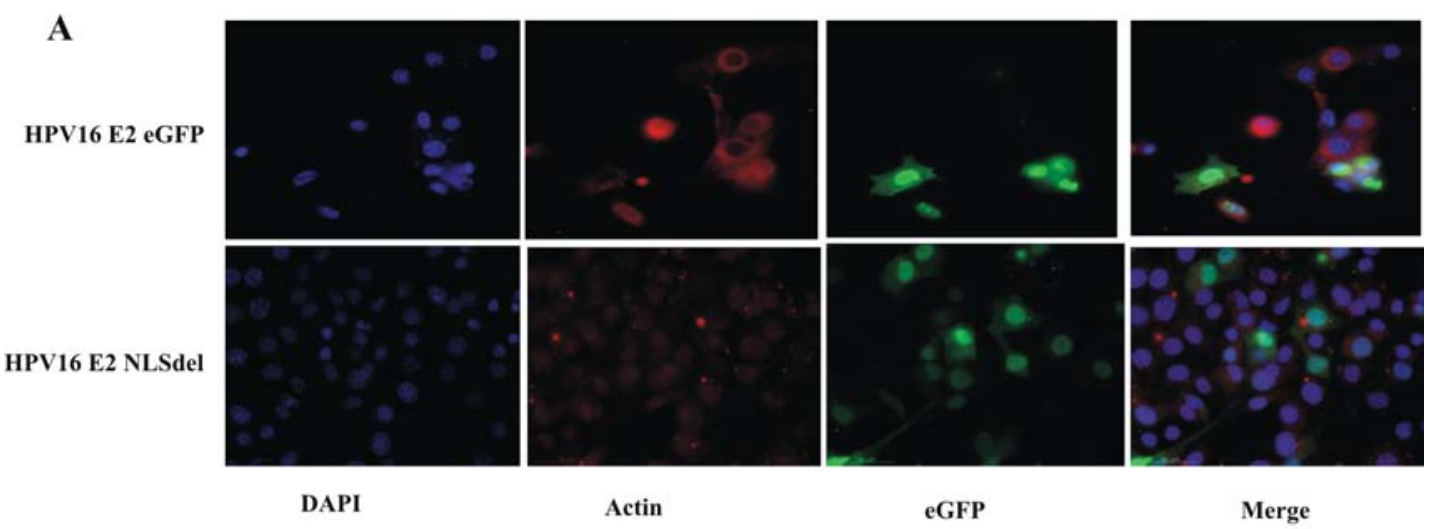

B

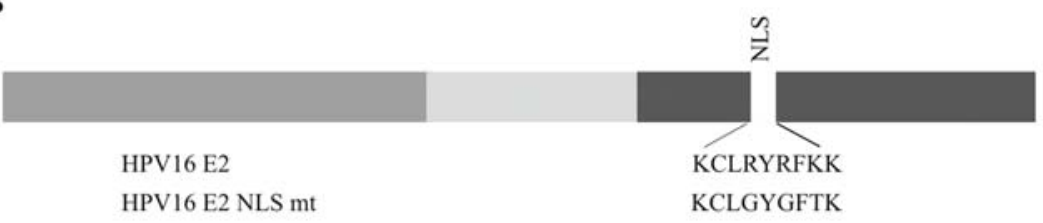

C

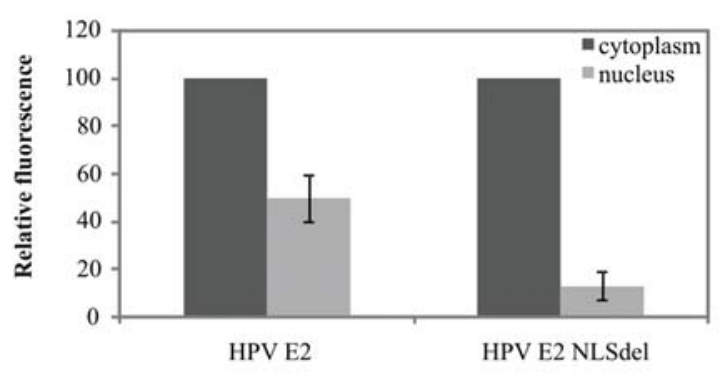

\section{D}

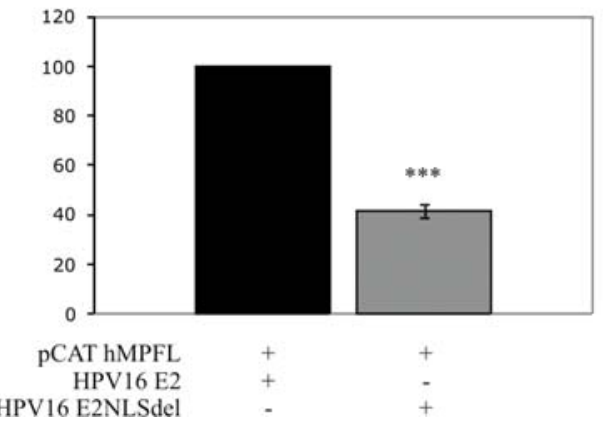

Figure 5. Effect of the deletion of the nuclear localisation signal present in HPV16 E2. (A) The localisation of the mutant as determined using an eGFP-fusion protein and immunofluorescence (blue, DAPI; red, actin; green, eGFP). Pictures were taken using a Zeiss AxioImager PlanAPOCHROMAT microscope (63x,1.4 oil), an AxioCam MRm R3, and AxioVision 4.7 software. (B) Schematic representation of the mutation introduced in order to eliminate the NLS. (C) ImageJ software was used to determine the correlation between whole cell fluorescence and nuclear fluorescence. Relative fluorescence ratios between nucleus and whole cells of an average of 10 transfected cells. (D) Effect of the NES mutant on the activation of the MMP-9 promoter as compared to the wildtype E2. ${ }^{*} \mathrm{P} \leq 0.05,{ }^{* *} \mathrm{P} \leq 0.01$ and ${ }^{* * *} \mathrm{P} \leq 0.005$ as obtained by Students' t-test. Results show mean value out of three experiments performed in triplicate.

tumors, c-Jun expression has been shown to correlate with the expression of the matrix metalloproteinase-1 and -9 (32). c-Jun negative ras-transformed cells lack many characteristics of Ras transformation including the ability to induce tumours in nude mice (33). Furthermore, ectopical expression of c-Jun in breast cancer cells resulted in a change in AP-1 composition and subsequent conversion into a more aggressive cell line and additionally also an increase in MMP-9 (34). Co-transfection of cells with the transactivation-deficient c-Jun mutant TAM67 leads to the integration of the TAM67 into newly forming AP-1 complexes which compete with functional AP-1 complexes for binding to the AP-1 binding motifs. Upon binding of a complex containing TAM67, transcription of the respective gene cannot progress and is abolished (35). TAM67 expression has already shown the importance of c-Jun in the growth of non-small cell lung cancer cells (36). Furthermore, TAM67 can abrogate an oncogenic phenotype in EpsteinBarr Virus (EBV) late-membrane protein-1 (LMP-1) positive nasopharyngeal carcinoma (37) and inhibits breast cancer growth in vivo and in vitro (38). Most interestingly for this study, TAM67 has been shown to block HPV16 E7-enhanced tumour progression in mice (39). Here, using co-transfection of TAM67, we were able to observe a highly significant inhibition of MMP-9 promoter activation by HPV16 E2. It can hence be concluded that c-Jun is a member of the transcriptionfactor complex binding to the MMP-9 promoters which is also supported by the above mentioned finding by Smith et al (34) that an overexpression of c-Jun results in an increase in MMP-9 expression. Furthermore, the highly significant inhibition detected could probably be due to the fact that TAM67 may also inhibit NF-кB (40).

An enhanced activation of ERK has been described in prostate (41), ovarian (42), and cervical cancers (43) among others. Its up-regulation is further known to be of importance in the phosphorylation and regulation of c-Jun (44). It has often been described that the human MMP-9 promoter is regulated by the activity of the mitogen-activated protein kinases. Most often this regulation is a result of the ability of MAP-kinases to induce transcription of members of the transcription factor complex AP-1 $(22,45)$. To investigate the 
role of the MAP-kinase members JNK, p38, and ERK, in the papillomavirus E2-mediated activation of the MMP-9 promoter, chemical inhibitors were added to the cells. In subsequent reporter-gene assays, a highly significant decrease in promoter activation could be detected in cells treated with PD098059, a MEK1-inhibitor (46). As it has previously been shown by our group $(15,20)$, this inhibitor was able to block CRPV E2-induced invasion in rabbit epithelial cells. Western blot analysis of the phosphorylation state of ERK in this study yielded evidence for phosphorylation of ERK and its downstream target ELK in human cells after transfection with the HPV16 E2 proteins. It has been shown that several viral proteins are involved in the regulation of MAPK pathways. The E5 protein of HPV16 is commonly known to activate ERK through its upregulation of EGF-receptor signalling $(28,47,48)$. The Karposi Sarcoma-Associated Herpesvirus (KSHV) induces AP-1 by activation of primarily ERK and JNK (49) similar to the hepatitis B Virus HBx protein, which also initiates AP-1 activity via ERK and JNK (50). The LMP-1 protein of EBV, which is also known to upregulate MMP-9, has been shown to influence a variety of cellular transcription factors such as NF- $\mathrm{KB}$ and AP-1 (51).

In a study by Blachon et al (13) it was shown that E2 of high-risk HPV-types is located within the nucleus and cytoplasm of an infected cell. It is able to move freely between the two compartments due to the presence of a nuclear export signal (NES) and a nuclear localisation sequence (NLS). Low-risk HPV E2 on the other hand is present solely within the nucleus. As opposed to high-risk E2, low-risk E2 proteins contain three NLSs but no NES. In their study, Blachon et al associated the localisation of the proteins with the induction of apoptosis as can be detected in high-risk HPV infection. Using HPV16 E2 mutants, we set out to investigate the role of E2 localisation on the protein's ability to activate the human MMP-9 promoter through the MEK1-ERK1/2/AP-1 signalling pathway. To confirm the localisation of the constructed mutants within the cell, the E2 protein and mutants were tagged with enhanced green fluorescent protein (eGFP). Introduction of either of the two additional NLS sites identified in low-risk HPV E2 into the HPV16 E2 sequence resulted in a mainly nuclear localisation of the protein. Activation of the human MMP-9 promoter as detected by reporter-gene assays was similar to that achieved by wild-type HPV16 E2.

As the predominantly nuclear localised protein was still able to activate the MMP-9 promoter, it could be hypothesised that the E2 protein induced MMP-9 expression relies on the localisation of the protein within the nucleus. Two methods were available in order to prove this theory. It is known that the nuclear import of the E2 protein occurs via the exportin-1 receptor (CRM1) which can be inhibited by addition of actinomycin D. However, the import of MAP-kinases into the nucleus occurs by a similar mechanism and hence inhibition of nuclear import may lead to non-interpretative results in the reporter-gene assays. The second option consisted of a deletion mutant of the NLS region of the E2 protein. Immunofluorescence of the created mutant confirmed a mainly cytoplasmic localisation of the protein. Reporter-gene assays showed a significant decrease in the activation potential of the mainly cytoplasmic E2 mutant. As the mutation introduced is not located within the transactivation domain of the
E2 protein, this decrease in activation cannot be accounted for by a loss in transactivation efficiency. Furthermore, as activation of the promoter cannot be due to direct binding of the E2 protein to the MMP-9 promoter, as no effect upon deletion of the E2 consensus sequence in the MMP-9 promoter was detected, this decrease in activation must be a result of the change in E2 localisation.

These results are interesting since it appears less likely that a cytoplasmic protein-protein interaction of E2 with protein kinases or phosphatases belonging to the ERK or p38-signal transduction pathway accounts for the activation of MMP-9 expression. It seems more conceivable that a protein-protein or protein-DNA interaction within the nucleus and consecutive de novo synthesis of a protein yet to be identified is critical for the activation of MMP-9 through MEK1-ERK1/2/AP-1.

We have shown in this study that high-risk HPV16 E2 activates ERK1/2, AP-1 and MMP-9, all major regulators during cancer progression (22). MMP-9 has been demonstrated to play a role during the process of local cancer invasion, extracellular matrix remodelling, and is associated with poor outcome in various epithelial cancers (52). AP-1 is a major regulator of several proteases that are involved in cancer invasion (19). Given that integration of viral DNA is less common in HNSCCs as compared with cervical cancers, we may speculate that also loss of E2 expression is less common in these tumours. This considered, E2 may have a role in cancer progression in HNSCCs. This idea is further supported by the fact that episomal localisation of viral DNA is more commonly found in advanced stages of HNSCCs (9).

In summary, in this report we demonstrate that HPV 16 E2 activates targets such as ERK1/2, AP-1 and MMP-9 but more importantly that this process is greatly enhanced by a nuclear localisation of the E2 protein. The mechanism of inducing MMP-9 expression does not rely on direct binding of E2 to the MMP-9 promoter but depends on the integrity of the transactivation domain of the viral protein (15). It is therefore less likely that a cytoplasmic protein-protein interaction between protein kinase/phosphatase members of the MEK1ERK1/2 pathway or p38 and HPV16-E2 accounts for the activation of MMP-9 expression. We hypothesise that the interaction with other proteins in the nucleus culminating in the transcriptional activation of a promoter and expression of a yet unknown protein activates the ERK signal transduction cascade, AP-1, and consequently MMP-9.

\section{Acknowledgements}

We would like to thank S. Schinkel and Dr S. Poppelreuther for their excellent technical support with the imaging. Special thanks go to K. Feldbauer. This study was supported by a DFG grant (Si 634-4/1, Si 634-5/1), and a Deutsche Krebshilfe e.V. grant (107689) to C. Simon and a DFG grant (IF 6/1-1/2) to T. Iftner

\section{References}

1. Bosch FX, Lorincz A, Munoz N, Meijer CJ and Shah KV: The causal relation between human papillomavirus and cervical cancer. J Clin Pathol 55: 244-265, 2002. 
2. Gillison ML, Koch WM, Capone RB, et al: Evidence for a causal association between human papillomavirus and a subset of head and neck cancers. J Natl Cancer Inst 92: 709-720, 2000.

3. Shamanin V, zur Hausen H, Lavergne D, et al: Human papillomavirus infections in nonmelanoma skin cancers from renal transplant recipients and nonimmunosuppressed patients. J Natl Cancer Inst 88: 802-811, 1996.

4. Giri I and Yaniv M: Structural and mutational analysis of E2 trans-activating proteins of papillomaviruses reveals three distinct functional domains. EMBO J 7: 2823-2829, 1988

5. Sakai H, Yasugi T, Benson JD, Dowhanick JJ and Howley PM: Targeted mutagenesis of the human papillomavirus type 16 E2 transactivation domain reveals separable transcriptional activation and DNA replication functions. J Virol 70: 1602-1611, 1996

6. Sedman T, Sedman J and Stenlund A: Binding of the E1 and E2 proteins to the origin of replication of bovine papillomavirus. J Virol 71: 2887-2896, 1997.

7. McBride AA, Romanczuk H and Howley PM: The papillomavirus E2 regulatory proteins. J Biol Chem 266: 18411-18414, 1991.

8. Klaes R, Woerner SM, Ridder R, et al: Detection of high-risk cervical intraepithelial neoplasia and cervical cancer by amplification of transcripts derived from integrated papillomavirus oncogenes. Cancer Res 59: 6132-6136, 1999.

9. Koskinen WJ, Chen RW, Leivo I, et al: Prevalence and physical status of human papillomavirus in squamous cell carcinomas of the head and neck. Int J Cancer 107: 401-406, 2003.

10. Desaintes C, Demeret C, Goyat S, Yaniv M and Thierry F: Expression of the papillomavirus E2 protein in HeLa cells leads to apoptosis. EMBO J 16: 504-514, 1997.

11. Massimi P, Pim D, Bertoli C, Bouvard V and Banks L: Interaction between the HPV-16 E2 transcriptional activator and p53. Oncogene 18: 7748-7754, 1999

12. Webster K, Parish J, Pandya M, Stern PL, Clarke AR and Gaston K: The human papillomavirus (HPV) 16 E2 protein induces apoptosis in the absence of other HPV proteins and via a p53-dependent pathway. J Biol Chem 275: 87-94, 2002.

13. Blachon S, Bellanger S, Demeret $C$ and Thierry F: Nucleocytoplasmic shuttling of high risk human Papillomavirus E2 proteins induces apoptosis. J Biol Chem 280: 36088-36098, 2005.

14. Jeckel S, Huber E, Stubenrauch F and Iftner T: A transactivator function of cottontail rabbit papillomavirus E2 is essential for tumor induction in rabbits. J Virol 76: 11209-11215, 2002.

15. Behren A, Simon C, Schwab RM, et al: Papillomavirus E2 protein induces expression of the matrix metalloproteinase- 9 via the extracellular signal-regulated kinase/activator protein-1 signaling pathway. Cancer Res 65: 11613-11621, 2005.

16. Davidson B, Goldberg I, Kopolovic J, et al: Expression of matrix metalloproteinase- 9 in squamous cell carcinoma of the uterine cervix-clinicopathologic study using immunohistochemistry and mRNA in situ hybridization. Gynecol Oncol 72 . 380-386, 1999

17. Ikebe T, Shinohara M, Takeuchi H, et al: Gelatinolytic activity of matrix metalloproteinase in tumor tissues correlates with the invasiveness of oral cancer. Clin Exp Metastasis 17: 315-323, 1999

18. Dumas V, Kanitakis J, Charvat S, Euvrard S, Faure M and Claudy A: Expression of basement membrane antigens and matrix metalloproteinases 2 and 9 in cutaneous basal and squamous cell carcinomas. Anticancer Res 19: 2929-2938, 1999.

19. Westermarck J and Kahari VM: Regulation of matrix metalloproteinase expression in tumor invasion. FASEB J 13: 781-792, 1999

20. Muhlen S, Behren A, Iftner T, Plinkert PK and Simon C: AP-1 and ERK1 but not p38 nor JNK is required for CRPV early protein 2-dependent MMP-9 promoter activation in rabbit epithelial cells. Virus Res 139: 100-105, 2009.

21. Bakiri L, Matsuo K, Wisniewska M, Wagner EF and Yaniv M: Promoter specificity and biological activity of tethered AP-1 dimers. Mol Cell Biol 22: 4952-4964, 2002.

22. Ozanne BW, Spence HJ, McGarry LC and Hennigan RF: Transcription factors control invasion: AP-1 the first among equals Oncogene 26: 1-10, 2007.

23. Downward J: Targeting RAS signalling pathways in cancer therapy. Nat Rev Cancer 3: 11-22, 2003

24. Harding A, Tian T, Westbury E, Frische E and Hancock JF: Subcellular localization determines MAP kinase signal output. Curr Biol 15: 869-873, 2005

25. Piao C, Jin M, Kim HB, et al: Ribonucleotide reductase small subunit p53R2 suppresses MEK-ERK activity by binding to ERK kinase 2. Oncogene (In press).
26. Cohen-Armon M, Visochek L, Rozensal D, et al: DNAindependent PARP-1 activation by phosphorylated ERK2 increases Elk1 activity: a link to histone acetylation. Mol Cell 25: 297-308, 2007.

27. Dong Z, Crawford HC, Lavrovsky V, et al: A dominant negative mutant of jun blocking 12-O-tetradecanoylphorbol-13-acetateinduced invasion in mouse keratinocytes. Mol Carcinog 19: 204-212, 1997.

28. Crusius K, Auvinen E and Alonso A: Enhancement of EGFand PMA-mediated MAP kinase activation in cells expressing the human papillomavirus type 16 E5 protein. Oncogene 15: 1437-1444, 1997.

29. Newberry EP, Willis D, Latifi T, Boudreaux JM and Towler DA: Fibroblast growth factor receptor signaling activates the human interstitial collagenase promoter via the bipartite Ets-Ap1 element. Mol Endocrinol 11: 1129-1144, 1997.

30. Rangatia J, Vangala RK, Singh SM, et al: Elevated c-Jun expression in acute myeloid leukemias inhibits C/EBPalpha DNA binding via leucine zipper domain interaction. Oncogene 22: 4760-4764, 2003.

31. Mathas S, Hinz M, Anagnostopoulos I, et al: Aberrantly expressed c-Jun and JunB are a hallmark of Hodgkin lymphoma cells, stimulate proliferation and synergize with NF-kappa B. EMBO J 21: 4104-4113, 2002.

32. Pacheco MM, Kowalski LP, Nishimoto IN and Brentani MM: Differential expression of c-jun and c-fos mRNAs in squamous cell carcinoma of the head and neck: associations with uPA, gelatinase B, and matrilysin mRNAs. Head Neck 24: 24-32, 2002 .

33. Johnson R, Spiegelman B, Hanahan D and Wisdom R: Cellular transformation and malignancy induced by ras require c-jun. Mol Cell Biol 16: 4504-4511, 1996.

34. Smith LM, Wise SC, Hendricks DT, et al: cJun overexpression in MCF-7 breast cancer cells produces a tumorigenic, invasive and hormone resistant phenotype. Oncogene 18: 6063-6070, 1999.

35. Grant S, Freemerman AJ, Birrer MJ, et al: Effect of 1-beta-Darabinofuranosylcytosine on apoptosis and differentiation in human monocytic leukemia cells (U937) expressing a c-Jun dominant-negative mutant protein (TAM67). Cell Growth Differ 7: 603-613, 1996.

36. Shimizu Y, Kinoshita I, Kikuchi J, et al: Growth inhibition of non-small cell lung cancer cells by AP-1 blockade using a cJun dominant-negative mutant. Br J Cancer 98: 915-922, 2008.

37. Jin X, Song X, Li L, et al: Blockade of AP-1 activity by dominant-negative TAM67 can abrogate the oncogenic phenotype in latent membrane protein 1-positive human nasopharyngeal carcinoma. Mol Carcinog 46: 901-911, 2007.

38. Liu Y, Ludes-Meyers J, Zhang Y, et al: Inhibition of AP-1 transcription factor causes blockade of multiple signal transduction pathways and inhibits breast cancer growth. Oncogene 21: 7680-7689, 2002.

39. Young MR, Farrell L, Lambert P, Awasthi P and Colburn NH: Protection against human papillomavirus type 16-E7 oncogeneinduced tumorigenesis by in vivo expression of dominantnegative c-jun. Mol Carcinog 34: 72-77, 2002.

40. Li JJ, Rhim JS, Schlegel R, Vousden KH and Colburn NH: Expression of dominant negative Jun inhibits elevated AP-1 and NF-kappaB transactivation and suppresses anchorage independent growth of HPV immortalized human keratinocytes. Oncogene 16: 2711-2721, 1998.

41. Zayzafoon M, Abdulkadir SA and McDonald JM: Notch signaling and ERK activation are important for the osteomimetic properties of prostate cancer bone metastatic cell lines. J Biol Chem 279: 3662-3670, 2004.

42. Al-Ayoubi A, Tarcsafalvi A, Zheng H, Sakati W and Eblen ST: ERK activation and nuclear signaling induced by the loss of cell/matrix adhesion stimulates anchorage-independent growth of ovarian cancer cells. J Cell Biochem 105: 875-884, 2008.

43. Branca M, Ciotti M, Santini D, et al: Activation of the ERK/ MAP kinase pathway in cervical intraepithelial neoplasia is related to grade of the lesion but not to high-risk human papillomavirus, virus clearance, or prognosis in cervical cancer. Am J Clin Pathol 122: 902-911, 2004.

44. Cirillo G, Casalino L, Vallone D, Caracciolo A, De Cesare D and Verde P: Role of distinct mitogen-activated protein kinase pathways and cooperation between Ets-2, ATF-2, and Jun family members in human urokinase-type plasminogen activator gene induction by interleukin-1 and tetradecanoyl phorbol acetate. Mol Cell Biol 19: 6240-6252, 1999. 
45. Ozanne BW, Spence HJ, McGarry LC and Hennigan RF: Invasion is a genetic program regulated by transcription factors. Curr Opin Genet Dev 16: 65-70, 2006.

46. Favata MF, Horiuchi KY, Manos EJ, et al: Identification of a novel inhibitor of mitogen-activated protein kinase kinase. J Biol Chem 273: 18623-18632, 1998.

47. Cartin W and Alonso A: The human papillomavirus HPV2a E5 protein localizes to the Golgi apparatus and modulates signal transduction. Virology 314: 572-579, 2003.

48. Crusius K, Rodriguez I and Alonso A: The human papillomavirus type 16 E5 protein modulates ERK1/2 and p38 MAP kinase activation by an EGFR-independent process in stressed human keratinocytes. Virus Genes 20: 65-69, 2000.

49. Xie J, Pan H, Yoo S and Gao SJ: Kaposi's sarcoma-associated herpesvirus induction of AP-1 and interleukin 6 during primary infection mediated by multiple mitogen-activated protein kinase pathways. J Virol 79: 15027-15037, 2005.
50. Benn J, Su F, Doria M and Schneider RJ: Hepatitis B virus HBx protein induces transcription factor AP-1 by activation of extracellular signal-regulated and c-Jun N-terminal mitogen-activated protein kinases. J Virol 70: 4978-4985, 1996.

51. Yoshizaki T, Sato H, Furukawa M and Pagano JS: The expression of matrix metalloproteinase 9 is enhanced by Epstein-Barr virus latent membrane protein 1. Proc Natl Acad Sci USA 95: 3621-3626, 1998

52. O-Charoenrat P, Rhys-Evans PH and Eccles SA: Expression of matrix metalloproteinases and their inhibitors correlates with invasion and metastasis in squamous cell carcinoma of the head and neck. Arch Otolaryngol Head Neck Surg 127: 813-820, 2001. 